\title{
Estimation of motion and parameters of heat transport from thermography
}

${ }^{1}$ Digital Image Processing, IWR, University of Heidelberg, Heidelberg, Germany

${ }^{2}$ Institut für Umweltphysik, University of Heidelberg, Heidelberg, Germany

\begin{abstract}
In this contribution a technique for measuring motion and parameters of temperature change locally in thermal image sequences will be presented. This leads to the estimation of highly accurate optical flow and parameters of heat transport, such as the constant of diffusivity or the matrix of anisotropic diffusion. Results of the computation are shown on a number of sample applications.
\end{abstract}

\section{Introduction}

Thermographic techniques are widely used for non-destructive testing of new materials. These methods have several advantages over other techniques in that they allow for an inspection of relative large surfaces in short time frames. At the same time they offer a robust and reliable detection of defects. Although presenting a number of advantages in the field of NDT, most current techniques are limited to static objects. The reason for this restriction is that motion is not taken into consideration. As an example one might want to look into Lock-In thermography. Here a time series is recorded and a Fourier transform performed at every pixel. Obviously, this technique only makes sense if it is assumed that the scene location mapped onto one pixel remains there during the duration over which the Fourier transform is computed. Otherwise the amplitude and phase information would be a mixture of those from different locations of an object or worse still, from different objects.

For a number of potential applications, the observed materials can not be made to remain still during image acquisition. This might be due to the movement of objects on a conveyor belt in industrial applications. Moreover, the observed effect in materials might be due to movement itself, such as the thermo-elastic effect. Here a temperature change is induced by compressing or depressing a material, which can be induced by bending it. By estimating the image velocity, motion compensation can be performed. This allows warping of thermal images, making pixels in subsequent frames to correspond to the same object location.

Due to physical transport processes, the objects under observation will change in temperature. This change has to be taken into account when estimating its motion. The transport of heat can usually be described by a differential equation, which turns out to be the diffusion equation in the case of heat transport due to conduction. By incorporating this model into the equation of motion, not only can the object velocity be estimated very accurately, but also the parameters of heat transport. In the following sections it will be demonstrated how image velocity and the constant of diffusion, or the in the case of anisotropic diffusion the whole matrix of diffusivity are accurately retrieved in a single estimation step. Since the estimate is performed on a small spatial temporal neighborhood of the image pixel of interest, both temporally and spatially highly resolved measurements are obtained. These estimates of the constant of diffusion are highly accurate and limited only by the frame rate and resolution of the infrared camera. 


\section{Deriving the optical Flow from Image Sequences}
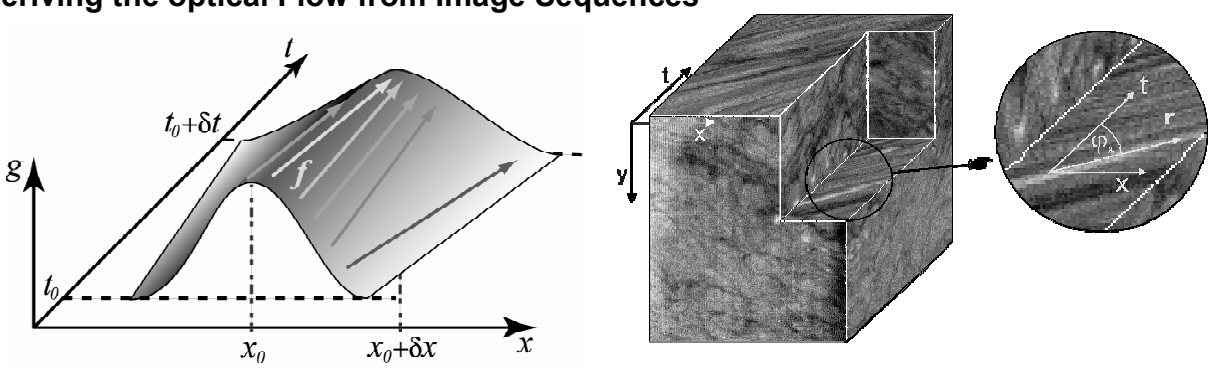

Figure 1: Illustration of the brightness change constraint equation. A one dimensional grey value distribution moves along the $x$-axis. In a constant brightness is violated due to a diffusion process. In $\boldsymbol{b}$ an actual IR Sequence is shown.

A very common assumption in optical flow computations is the brightness change constraint equation (BCCE) [1]. It is assumed that the image brightness of a scene point remains constant in a spatio-temporal neighborhood. That is the image intensity $g$ at the location $(x, y)$ at time $t$ stays the same in a time interval $\partial t$ during which a translation by $(\partial x, \partial y)$ took place. This brightness constancy model can be formulated as

$$
g(x+\partial x, y+\partial y, t+\partial t)=g(x, y, t) .
$$

[2] proved that this assumption holds provided that no illumination changes are present and the surface of the object are Lambertian in nature.

Clearly this assumption does not hold in most real world situation, which is especially true for thermographic applications. In thermography, the temperature distribution of scene objects is visualized. Frequently the temperature of these objects changes due to diffusion processes, leading to a change in image intensities. Obviously this brightness change is not connected to any motion, thus violating the assumption of brightness constancy. This effect is illustrated in figure 1. Here isobrightness lines are not corresponding to the movement any more. To accommodate this fact an extension to the used conservation law has to be formulated. The use of a linear model with a multiplier and offset term has been suggested [3]. Here we follow $[4 ; 5]$ in reformulating the brightness change as a linear partial differential equation.

The brightness of a moving pattern is allowed to change according to an analytical function $h$ where $h(g(\vec{x}), \vec{b})$ is a scalar invertible transformation with the q-dimensional parameter vector $\vec{b}=\left(b_{1}, \ldots, b_{q}\right)$ and the identity element $h(g(\vec{x}), 0)=g(\vec{x})$. The total derivative of the grey value is then given by

$$
g_{t}+(\vec{u} \nabla) g=f\left(g^{\prime}(\vec{x}), \vec{b}\right) \text {, with } f\left(g^{\prime}(\vec{x}), \vec{b}\right)=\frac{d}{d t} h\left(g^{\prime}(\vec{x}), \vec{b}\right) \text {. }
$$

With the formulation of the generalized brightness change constraint equation (2) it is now possible to estimate reliable optical flow in the presence of intensity 
changes. Moreover, in scientific applications the image intensity change might be due to physical phenomena, the parameters of which can be estimated reliably from equation (2). This type of equation can be solved locally in an over determined system of equations in a total least squares sense [6]. This is done by assuming constant parameters in a local neighborhood and pooling the constraints for each pixel. Examples of this new field of problems will be presented in the next section.

In equation (2) the motion vector is modeled as that of a rigid body. In fluid motion this might not always be adequate. Often the velocity field $\vec{u}$ is represented in a local neighborhood by an affine flow model $[7 ; 8]$ :

$$
\vec{u}=\vec{t}+\mathbf{A} \vec{x}=\left(\begin{array}{l}
t_{1} \\
t_{2}
\end{array}\right)+\left(\begin{array}{ll}
a_{1} & a_{2} \\
a_{3} & a_{4}
\end{array}\right)\left(\begin{array}{l}
x \\
y
\end{array}\right) .
$$

Here $t$ represents the center velocity of the neighborhood. The parameters of the affine transformation matrix $\mathrm{A}$ can be used directly to estimate convergence and divergence of the flow field. This model is a superposition of uniform motion, rotation, dilation and shear. It is of great interest in applications such as the estimation of the flow field at the sea surface, where divergences are important parameters in sea surface gas exchange.

\section{Applications}

In the preceding sections a framework was presented for simultaneously estimating the parameters of optical flow and brightness changes. These estimations are highly relevant for a number of applications, some of which will be presented in the following.

\subsection{Estimating the total derivative at the air water interface}

The total derivative of the sea surface temperature with respect to time $\mathrm{d} T / \mathrm{d} t$ is an important quantity in air-sea heat exchange. From this derivative the net heat flux can be derived [5]. It is given by $d T / d t=\partial T / \partial t+(\vec{u} \nabla) T$ where $T$ is the sea surface temperature and $\vec{u}=\left(u_{1}, u_{2}\right)^{\mathrm{T}}$ the surface flow. By comparing this equation with Equation (2) it becomes apparent, that $\mathrm{d} T / \mathrm{d} t$ can be modeled by a linear source term, that is $g_{t}+u_{1} g_{x}+u_{2} g_{y}=c$.
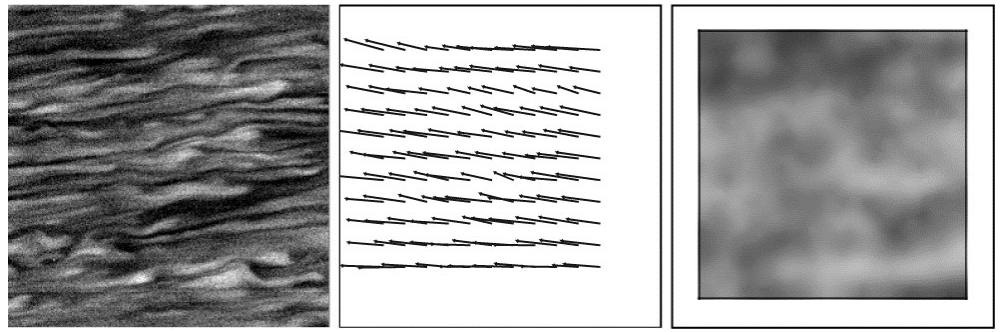

Figure 2: An image from a sequence with the corresponding 2D optical flow and the total derivative of the temperature. 
The problem is solved by extending this constraint equation onto a local neighborhood, yielding one equation per pixel. Solving this system of equations in a total least squares sens, the right singular vector $\vec{e}=\left(e_{1}, e_{2}, e_{3}, e_{4}\right)^{\mathrm{T}}$ to the smallest singular value $\lambda$ of the data matrix $D$ in

$$
\left(\begin{array}{cccc}
-1 & g_{x, 1} & g_{y, 1} & g_{t, 1} \\
-1 & g_{x, 2} & g_{y, 2} & g_{t, 2} \\
\vdots & \vdots & \vdots & \vdots \\
-1 & g_{x, n} & g_{y, n} & g_{t, n}
\end{array}\right) \cdot\left(\begin{array}{c}
c \\
u_{1} \\
u_{2} \\
1
\end{array}\right)=D \cdot p=0
$$

represents the sought solution to the problem. The full parameter field for one image of a sequence is shown in figure 2. The total derivative $d T / d t=c=e_{1} / e_{4}$ and the surface flow $\vec{u}=\left(u_{1}, u_{2}\right)^{\mathrm{T}}=\left(e_{2} / e_{4}, e_{3} / e_{4}\right)^{\mathrm{T}}$ and the can thus be estimated.

\subsection{D flow with affine parameterization}

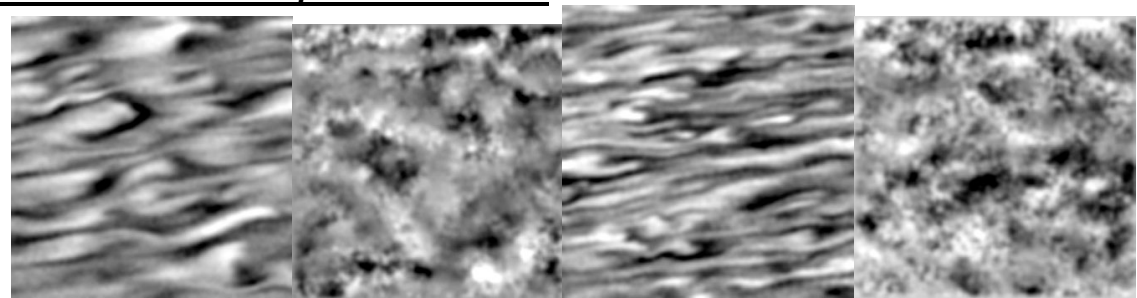

Figure 3: The divergence estimated for the infrared sequences. In a an image recorded at a wind speed of $2 \mathrm{~m} / \mathrm{s}$ can be seen with the corresponding divergence in $\boldsymbol{b}$. The same is shown in $\boldsymbol{c}$ and $\boldsymbol{d}$ for a wind speed of $4.2 \mathrm{~m} / \mathrm{s}$.

In section 2 the extension of the constant flow model to affine model was introduced. In terms of modelling actual physical processes the affine flow model is highly relevant. In a number of processes the affine matrix $A$ from Equation (3) is an important quantity. In oceanographic applications, divergence and convergence at the sea surface present an important parameter in air-water gas and heat exchange. At the sea surface a source term is still required as stated in the previous section. The set of equations solving this optical flow problem can be formulated as

$$
\left(\begin{array}{cccccccc}
-1 & g_{x, 1} & g_{y, 1} & x_{1} g_{x, 1} & y_{1} g_{x, 1} & x_{1} g_{y, 1} & y_{1} g_{y, 1} & g_{t, 1} \\
-1 & g_{x, 2} & g_{y, 2} & x_{2} g_{x, 2} & y_{2} g_{x, 2} & x_{2} g_{y, 2} & y_{2} g_{y, 2} & g_{t, 2} \\
\vdots & \vdots & \vdots & \vdots & \vdots & \vdots & \vdots & \vdots \\
-1 & g_{x, n} & g_{y, n} & x_{n} g_{x, n} & y_{n} g_{x, n} & x_{n} g_{y, n} & y_{n} g_{y, n} & g_{t, n}
\end{array}\right) \cdot\left(\begin{array}{c}
c \\
u_{1} \\
u_{2} \\
a_{1} \\
a_{2} \\
a_{3} \\
a_{4} \\
1
\end{array}\right)=D \cdot p=0 .
$$

As explained in section 2 the neighborhood center velocity is given by $\vec{u}=\left(u_{1}, u_{2}\right)^{\mathrm{T}}$. Results of this computation are presented in figure 3 . 
In botanical application, where the growth of plant leaves or roots is of interest, the divergence can be estimated directly and the regions of growth quantified [9]. Due to constant illumination the source term $c$ in equation (5) can be removed together with the first column of the matrix, resulting in $D \in \mathbb{R}^{n \times 7}$ and $p \in \mathbb{R}^{7}$.

It should be emphasized that because of the projective properties of the imaging system movement of objects in the line of sight of the camera also lead to an apparent divergence. The distance of the object to the camera has to be kept constant, or the change thereof has to be measured as well, if physically meaningful divergences are to be deducted from the estimation.

\subsection{Flow with Isotropic Diffusion}

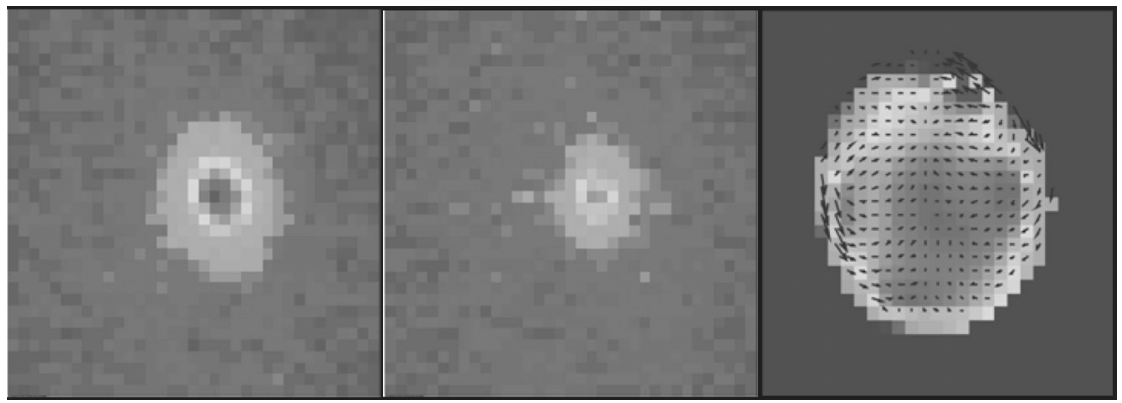

Figure 4: Isotropic diffusion of heat in a polymer heated with a laser. In $\mathbf{a}$ and $\boldsymbol{b}$ two frames of the sequence are shown and in c the computed diffusivity and motion.

Isotropic diffusion is a phenomenon commonly associated with heat. The corresponding set of equations is given by

$$
\left(\begin{array}{cccc}
-\Delta g_{1} & g_{x, 1} & g_{y, 1} & g_{t, 1} \\
-\Delta g_{2} & g_{x, 2} & g_{y, 2} & g_{t, 2} \\
\vdots & \vdots & \vdots & \vdots \\
-\Delta g_{n} & g_{x, n} & g_{y, n} & g_{t, n}
\end{array}\right) \cdot\left(\begin{array}{c}
D \\
u_{1} \\
u_{2} \\
1
\end{array}\right)=\mathbf{D} \cdot \mathbf{p}=0 .
$$

where $D$ is the constant of diffusion and $\Delta g_{i}=\left(\partial^{2} g_{i} / \partial x^{2}+\partial^{2} g_{i} / \partial y^{2}\right)$ is the Laplace operator of the i-th pixel.

In figure 4 an example of such a process is presented. A slab of polymer is heated up by a laser. The heating is turned off an the heat transported in the polymer by heat diffusion. The estimated constant of diffusivity can be compared to that of the polymere.

\subsection{D Flow with Anisotropic Diffusion}

The case of a direction dependant two-dimensional diffusion can be modeled by $g_{x} \cdot u_{1}+g_{y} \cdot u_{2}+g_{t}-\nabla(D \cdot \nabla) g=0$, with the anisotropic diffusion tensor D. This tensor is given by

$$
D=\left(\begin{array}{ll}
d_{00} & d_{01} \\
d_{10} & d_{11}
\end{array}\right)=\left(\begin{array}{ll}
d_{00} & d_{10} \\
d_{10} & d_{11}
\end{array}\right),
$$




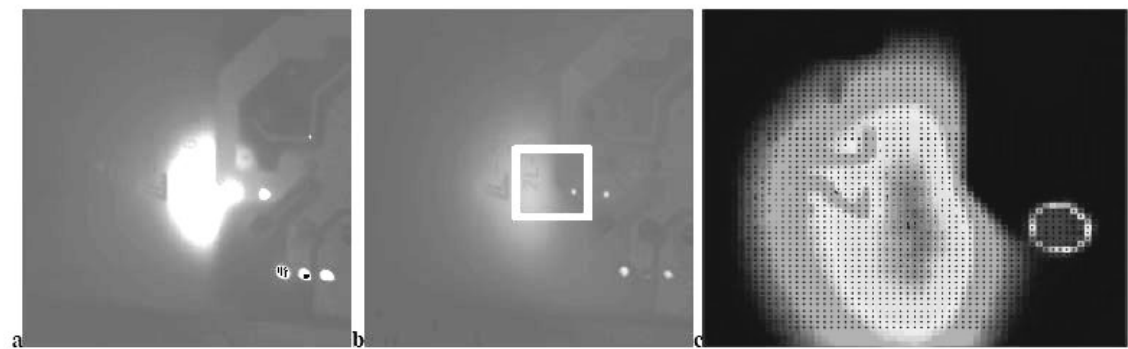

Figure 5: In a a printed circuit board is heated up. Image velocity and the diffusivity matrix are computed for the highlighted region in $\boldsymbol{b}$, the results are displayed in $\boldsymbol{c}$.

where use was made of the fact that the diffusion tensor $D$ is a symmetric tensor. Inserting this expression into the constraint equation leads to:

$$
\left(\begin{array}{cccccc}
g_{x, 1} & g_{y, 1} & -g_{x x, 1} & -g_{y y, 1} & -2 g_{x y, 1} & g_{t, 1} \\
g_{x, 2} & g_{y, 2} & -g_{x x, 2} & -g_{y y, 2} & -2 g_{x y, 2} & g_{t, 2} \\
\vdots & \vdots & \vdots & \vdots & \vdots & \vdots \\
g_{x, n} & g_{y, n} & -g_{x x, n} & -g_{y y, n} & -2 g_{x y, n} & g_{t, n}
\end{array}\right) \cdot\left(\begin{array}{c}
u \\
v \\
d_{00} \\
d_{11} \\
d_{10} \\
1
\end{array}\right)=D \cdot p=0 .
$$

An example of anisotropic diffusion of heat is shown in figure 5. Here a printed circuit board is locally heated up. Due to different materials making up the compound, heat is transported differently at different parts of the board.

\section{References}

[1] Horn BKP, Schunk B. Determining Optical Flow. Artificial Int. 17, 185-204. '81.

[2] Verri A, Poggio T. Motion field and optical flow: Qualitative Properties. IEEE Transactions on Pattern Analysis and Machine Intelligence 11[5],490-498. '89.

[3] Negahdaripour S, Yu CH. A Generalized Brightness Chane Model for Computing Optical Flow. Int. Conference in Computer Vision. 2-7. '93.

[4] Haußecker H, Fleet DJ. Computing Optical Flow with Physical Models of Brightness Variation. PAMI 23[6], 661-673. 2001.

[5] Garbe CS, Spies H, Jähne B. Estimation of surface flow and net heat flux from infrared image sequences. J. Math. Imaging and Vision 19[3], 159-174. 2003.

[6] Van Huffel S, Vandewalle J. The Total Least Squares Problem: Computational Aspects and Analysis. SIAM; 1991.

[7] Black MJ, Jepson AD. Estimating optical flow in segmented images using variable-order parametric models. PAMI 18[10], 972-986. '96.

[8] Fleet DJ. Measurement of Image Velocity: Kluwer Academic; 1992.

[9] Garbe CS, Schurr U, Jähne B. Thermographic measurements of plant leaves. Maldague, X. P. and Rozlosnik, A. E. SPIE. 4710, 407-416. 2002. 